\title{
Does lymph node morphology using ultrasound reflect aetiology? A pictorial essay, part II, malignant lymphadenopathy
}

\author{
Corinna Trenker ${ }^{1,2}$, Christian Görg ${ }^{2}$, Alois Hollerweger ${ }^{3}$, Christian Jenssen ${ }^{4}$, Yi Dong ${ }^{5}$, \\ Xin-Wu Cui ${ }^{6}$, Christoph F. Dietrich ${ }^{7}$
}

\begin{abstract}
${ }^{1}$ Department of Haematology, Oncology and Immunology, University Hospital Giessen and Marburg, Philipps University Marburg, Baldingerstraße, Marburg, Germany, ${ }^{2}$ Interdisciplinary Center of Ultrasound, University Hospital Giessen and Marburg, Philipps University Marburg, Baldingerstraße Marburg, Germany, ${ }^{3}$ Department of Radiology, Hospital Barmherzige Brüder, Salzburg, Austria, ${ }^{4}$ Department of Internal Medicine, Krankenhaus Märkisch-Oderland Strausberg/Wriezen and Brandenburg Institute for Clinical Ultrasound, Medical University "Theodor Fontane" Neuruppin, Germany, ${ }^{5}$ Department of Ultrasound, Zhongshan Hospital, Fudan University, Shanghai, China, ${ }^{6}$ Department of Medical Ultrasound, Tongji Hospital, Tongji Medical College, Huazhong University of Science and Technology, Wuhan, Hubei, China, ${ }^{7}$ Department Allgemeine Innere Medizin (DAIM), Kliniken Hirslanden Beau Site, Salem und Permancence, Bern, Switzerland
\end{abstract}

\footnotetext{
Abstract

The evaluation of lymph nodes (LN) using ultrasound requires a high level of clinical and sonographic competence. This "pictorial essay" is intended to illustrate eye-catching examples of relevant "clinical-sonographic visual diagnoses" of LNs. We provide typical images and take home messages of eye-catching features to illustrate the featured publications.

The first part included the introduction into the subject, indications for US examination and the widely accepted recognized features for LN characterization. The key features for the differential diagnosis of suspected lymphadenopathy and benign lymphadenopathy are illustrated. In the second part eye-catching features of malignant lymphadenopathy and the differential diagnosis of carcinoma and lymphoma are shown.
}

Keywords: ultrasound; neoplasia; hematology; lymph node

\section{Introduction}

In this second part eye-catching features of malignant lymphadenopathy and the differential diagnosis of carcinoma and lymphoma are shown. In general, LN metastases of solid tumours have to be differentiated from malignant lymphatic LN infiltrations [1-7].

The typical features of benign lymphadenopathy include the preserved architecture and painful swelling

Received 02.04.2020 Accepted 22.05.2020

Med Ultrason

2020, Vol. 22, No 4, 476-484

Corresponding author: Prof. Dr. med. Christoph F. Dietrich, MBA

Department of Internal Medicine (DAIM)

Kliniken Hirslanden Bern, Beau Site,

Salem and Permanence, Schänzlihalde 11, 3031 Bern, Switzerland

E-mail: c.f.dietrich@googlemail.com
[7,8] but indolent non-Hodgkin lymphoma may also show preserved vessel architecture, whereas Hodgkin's disease often demonstrates loss of $\mathrm{LN}$ architecture at an early stage [9].

The typical features of lymphoma, in addition to the eye-catching feature of splenomegaly, are multiplicity of painless echo-poor lymph nodes in chains arranged around the course of larger vessels without a specific relation to cancer organs. The LNs lose their architecture and may appear cystic. Similarly, tuberculosis has to be taken into account with focal lymph node lesions and necrotic tissue [10-15]. It has to be kept in mind that testicular and also ovarian lymph node metastasis may follow a similar pathway around the aorta and inferior vena cava. The lymph node vascularity of metastatic LNs is often similar to the vessel characteristics of the primary tumour. Hypervascular LNs are typically found in LN 
metastases of renal cell cancer, neuroendocrine, thyroid, breast and ovarian cancer [16-19].

As already mentioned in part one, regional cortical thickening, especially in otherwise pathological appearing round shaped lymph nodes, is a sign of circumscript malignant LN infiltration, especially seen in highly differentiated metastatic infiltration and also in less aggressive Hodgkin's and non-Hodgkin's disease [9,20-23]. The circumscript infiltration can be explained by malignant cells entering via afferent vessels with neoplastic growth. At this site peripheral enhancement of contrast agents with less central enhancement is indicative of malignant infiltration [24-27]. It is of interest that central portions of LNs may be less effectively treated by systemic chemotherapy or immunomodulating treatment [28-31]. In contrast, a regular vascular architecture and branching and enhancement of vessels in a centrifugal direction indicates benign lymphadenopathy.
Distorted vascular branching representing vessel amputation and increased neoplastic vessel density are observed more often in cancers with a poor prognosis. It is well known that metastatic nodes have a higher resistive index (RI) compared to inflammatory LNs. When intranodal LN pressure rises, the arterial RI rises as well if the capsule is intact [32]. In these circumstances typically only arteries can be detected by Doppler techniques as small veins may be compressed by high intranodal pressure [25,26,33-35]. The neoplastic vessels and the compressed veins may cause ischemia stimulating further neoplastic reactions. In later stages transcapsular arteriovenous shunts may be predominant with RI in arteries lower than in earlier stages [32,36]. Due to capsule infiltration high pressure interstitial fluid may leak out of the LN causing perilymphatic oedema. Due to the loss of pressure the neoplastic vessel architecture may become visible again.

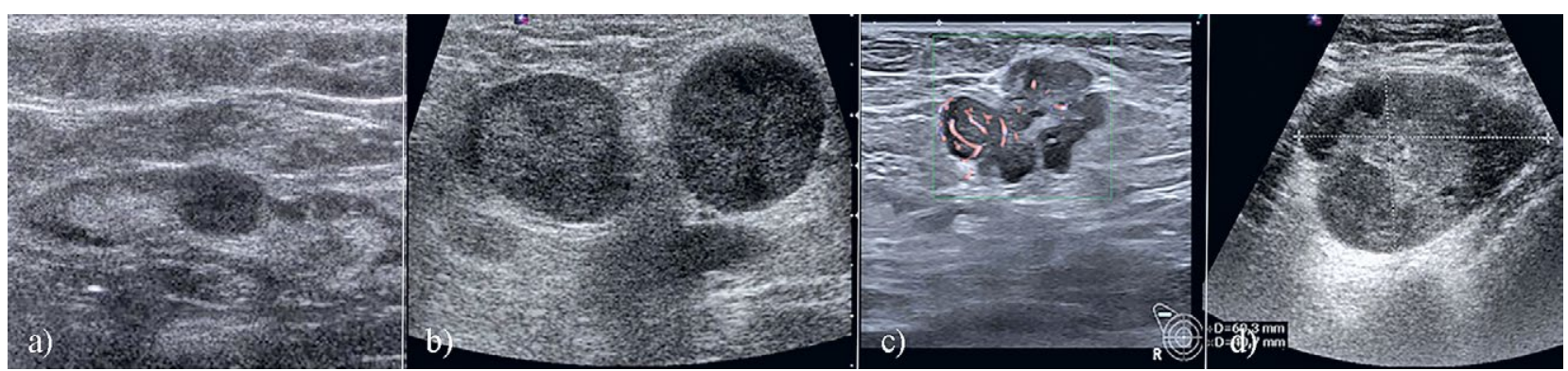

Fig 1. Different B-mode and colour Doppler patterns of metastatic LNs. A non-enlarged LN with a round echo-poor nodule within the inguinal LN in the follow up of a malignant melanoma. The LN was suspected for LN metastases (confirmed by surgery) (a). Two round and hypoechoic LNs visible in the inguinal region 2 years after cervical cancer surgery. US-guided biopsy confirmed LN metastases (b). Color Doppler ultrasound shows an enlarged hypoechoic LN with lobulated shape and irregular vessels in a patient with breast cancer. LN metastasis was confirmed by surgery (c). A marked enlarged axillary LN with heterogeneous structure and cystic areas in a case of metastasis from malignant melanoma (d). Take home message: a non-enlarged LN with a round echo-poor nodule, enlarged hypoechoic round LNs, enlarged LNs with irregular margins and chaotic vessel architecture or enlarged LNs with heterogeneous structure and necrotic areas is an eye-catching feature for LN metastasis. In patients with skin neoplasia and specifically melanoma located distal to the elbow or the knee, the cubital and/or popliteal fossae should be also examined.
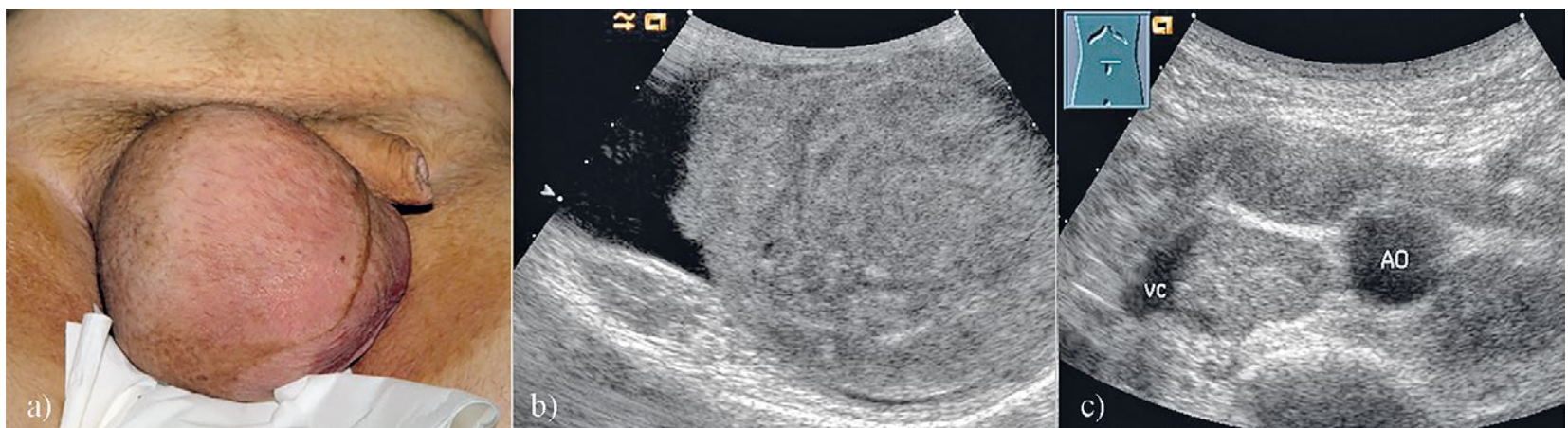

Fig 2. Male patient presents with palpable right-sided testicular enlargement (a). B-mode ultrasound shows a large and heterogeneous testis suspected to be testicular carcinoma (confirmed by surgery) (b). In the paraaortic area of the renal arteries enlarged LNs were observed suspected to be metastases (c). Take home message: the presence of enlarged LNs in the paraaortic area of the renal arteries is an eye-catching feature for LN metastasis from testicular carcinoma. 
In lymphomas, the tumour neovascularization also depends on their aggressiveness or indolence. High microvessel density is more often seen in aggressive entities and stages with a poor prognosis if no treatment options are available [37-40]. Even if both, lymphoma and meta- tasis, present characteristic B-mode and Colour Doppler (CD) imaging, the diagnosis cannot be achieved only by ultrasound. Patients symptoms and past medical history are important and finally the histological confirmation of the lymph node is crucial.
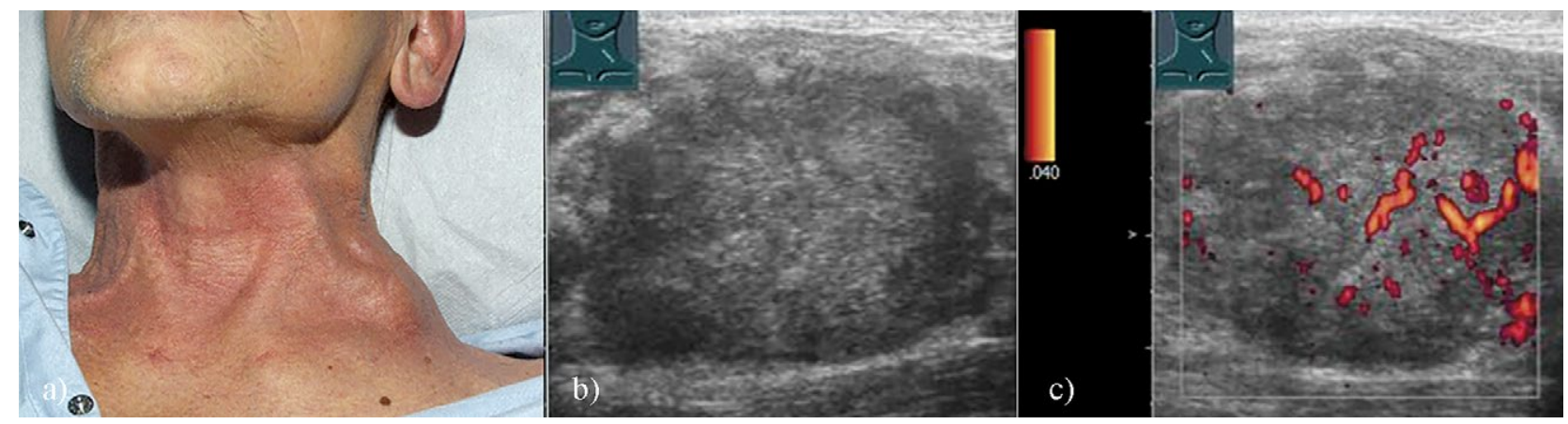

Fig 3. Patient presents with a palpable hard lesion in the left neck triangle, suspected to be an enlarged LN (a). B-mode imaging shows a LN in the "Virchow gland" location (b). The lesion showed isolated vessels suspected to be due to malignancy (c). US guided core needle biopsy confirmed the diagnosis of a LN metastasis from prostate cancer. Take home message: the presence of an enlarged $L N$ in the "Virchow gland" location with isolated vessels is an eye-catching feature for LN metastasis.

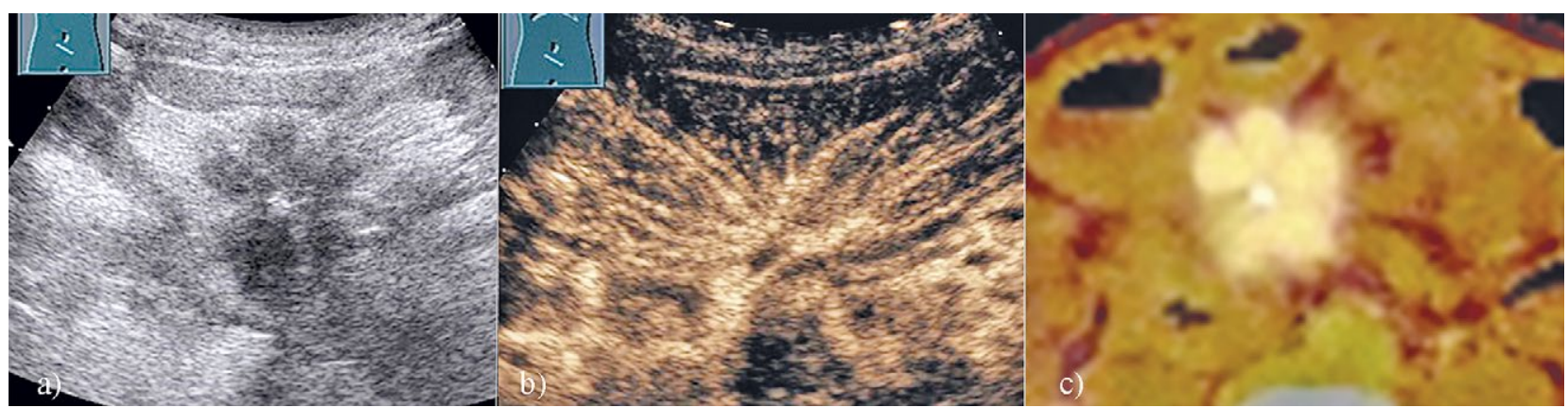

Fig 4. Patient presents with history of a NET, abdominal pain and subileus. In B-mode imaging, a mesenteric LN metastasis, localized around the superior mesenteric artery was seen, which was pulling on intestinal loops suggestive of a desmoplastic reaction (a). CEUS shows mesenteric vessels with a star-shaped appearance due to pulling of the bowel loops (b). The somatostatin receptor PET-CT proves diagnosis of mesenteric LN metastasis of NET (c). Take home message: the presence of enlarged LN pulling on intestinal loops suggestive of a desmoplastic reaction is an eye-catching feature for LN metastasis of NETs.
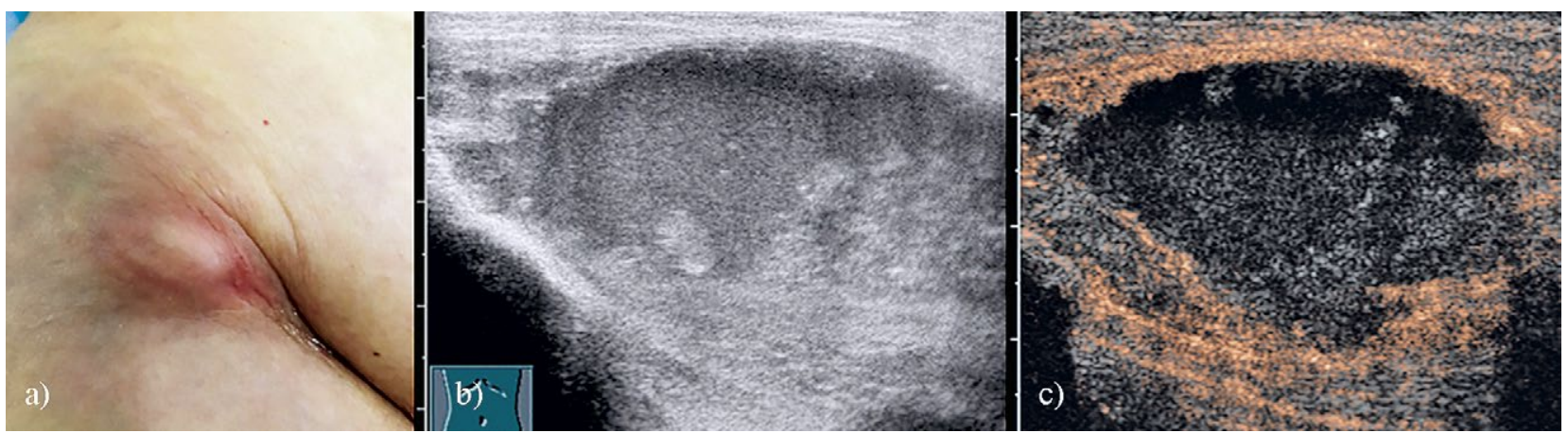

Fig 5. Patient presents with a painful palpable tumour in the right inguinal region (a). B-mode imaging shows a complex LN formation with necrotic areas (b). CEUS shows just a marginal contrast media uptake of the LN (c). The US guided core needle biopsy revealed the diagnosis of metastases from a squamous cell carcinoma. Take home message: the presence of enlarged heterogeneous LNs in the inguinal region with a marginal hyperenhancement on CEUS is an eye-catching feature for LN metastasis from anal or vulvar carcinoma. 


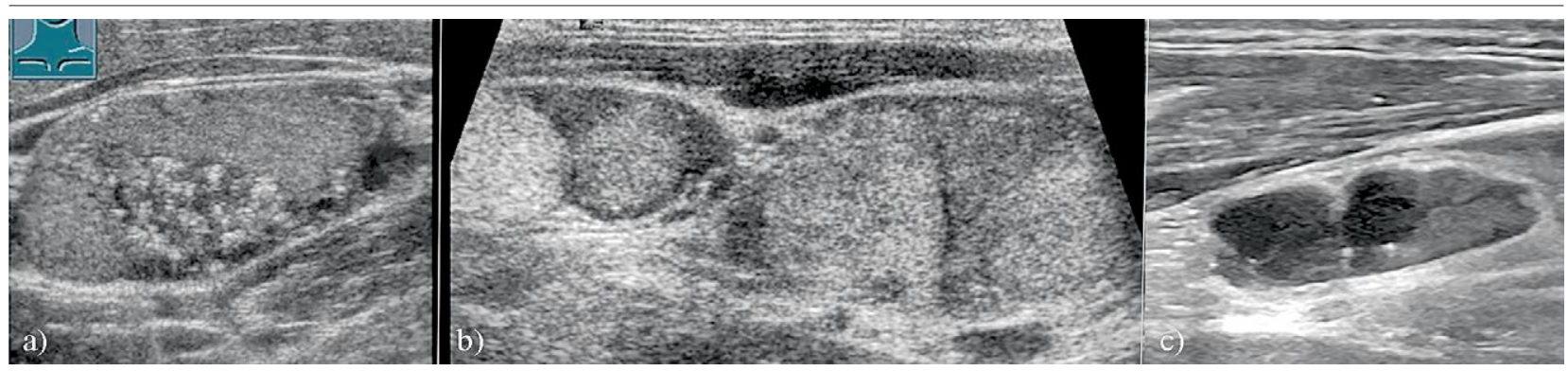

Fig 6. Patient presents with palpable tumour on the left side of the neck suspected to be an enlarged LN secondary to thyroid carcinoma. Longitudinal and cross-section B-mode imaging shows a complex LN with multiple small calcifications. US guided core needle biopsy revealed the diagnosis of a metastases from a thyroid carcinoma (a). Hyperechoic LNs on the right side adjacent to the large vessels and a predominantly cystic LN on the left side with some microcalcifications are further examples of metastases of papillary thyroid cancer (b,c). Take home message: the presence of enlarged heterogenous LNs with multiple small calcifications, partly cystic LNs or hyperechoic LNs is an eye-catching feature for neck LN metastasis from thyroid carcinoma.
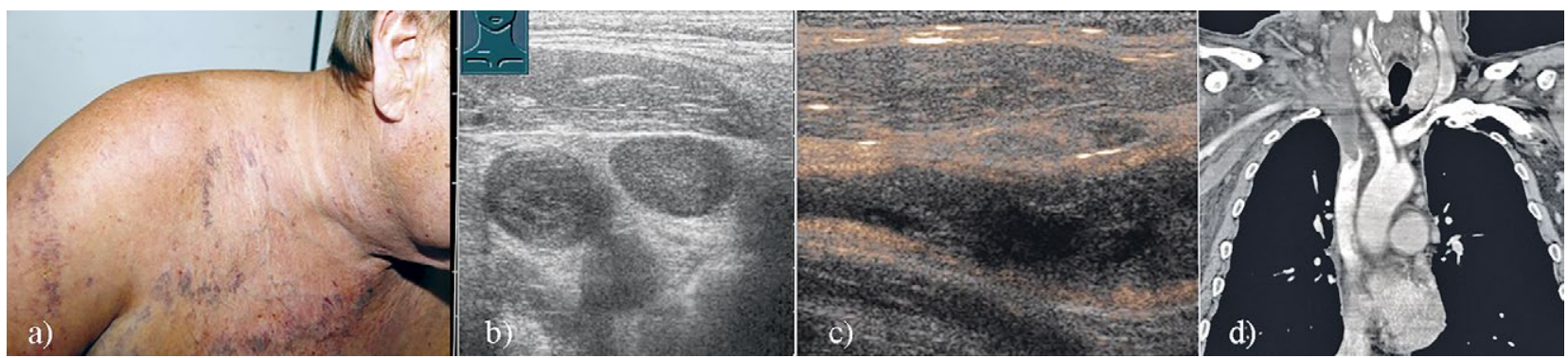

Fig 7. Patient presents with varicose skin vessels and acute shoulder, arm- and facial swelling suggestive of a superior vena cava obstruction (a). B-mode imaging shows a venous thrombosis and a pathological LN (b). CEUS confirms the diagnosis of thrombosis (c). US guided core needle biopsy gives the diagnosis of metastases from bronchial carcinoma. CT shows the thrombosis of the superior vena cava (d). Take home message: the presence of an enlarged pathological LN near venous thrombosis is an eye-catching feature for LN metastasis.

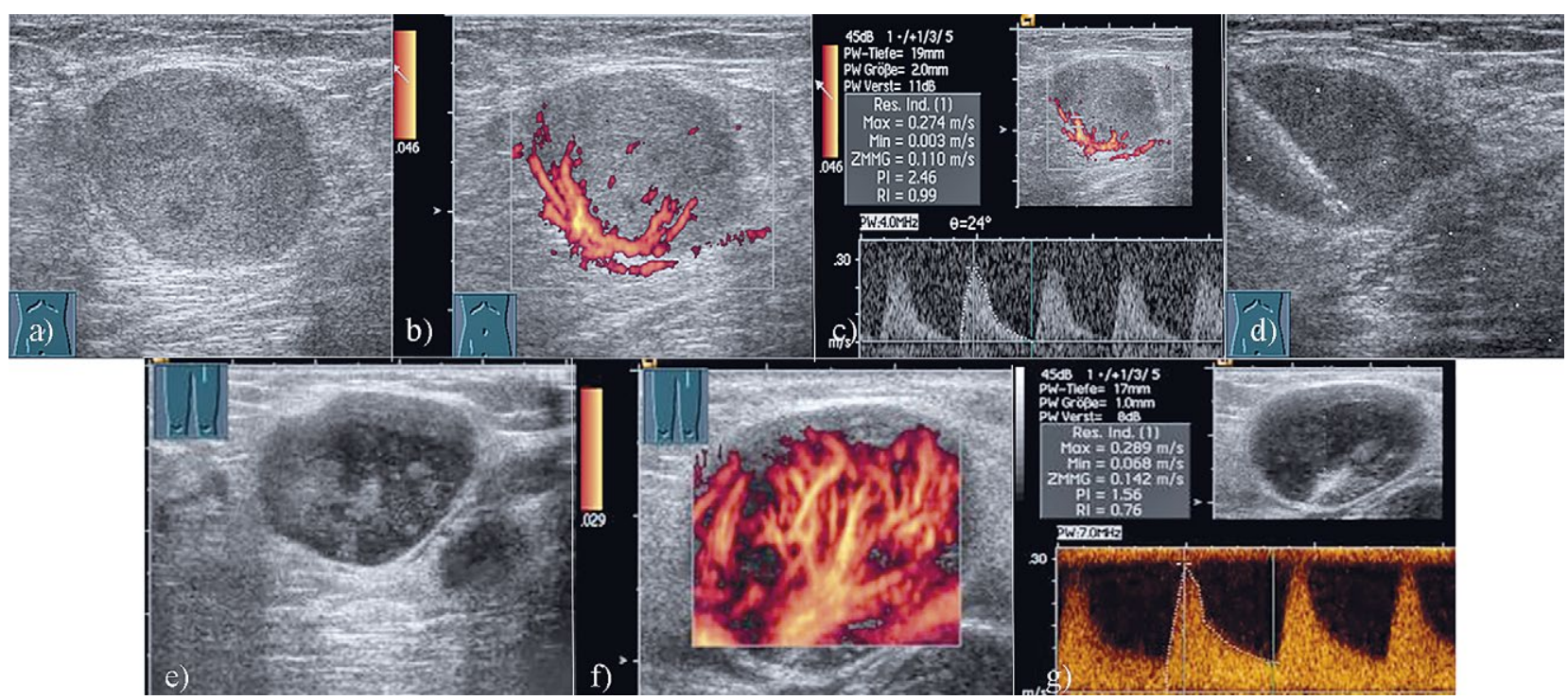

Fig 8. Patient presents with palpable nodes in the right inguinal region with previous surgery for rectal cancer. B-mode imaging shows oval echoic homogeneous nodes (a). CDS delineates an irregular peripheral vascular pattern (b). A high RI (0,99) was measured in these irregular arteries (c). US guided core needle biopsy revealed the diagnosis of lymph node metastasis from rectal cancer (d). For comparison an enlarged hypoechoic heterogeneous left sided inguinal lymph node (e) with hilar type vascular pattern (f) with regularly branching of lymph node vessels and a lower RI $(0,76)(\mathrm{g})$ measured in non-Hodgkin lymphoma is shown. Take home message: the presence of enlarged hypoechoic LN with a peripheral type vascular pattern and high RI are eye-catching features of lymph node cancer metastasis, but histologic examination is essential. 


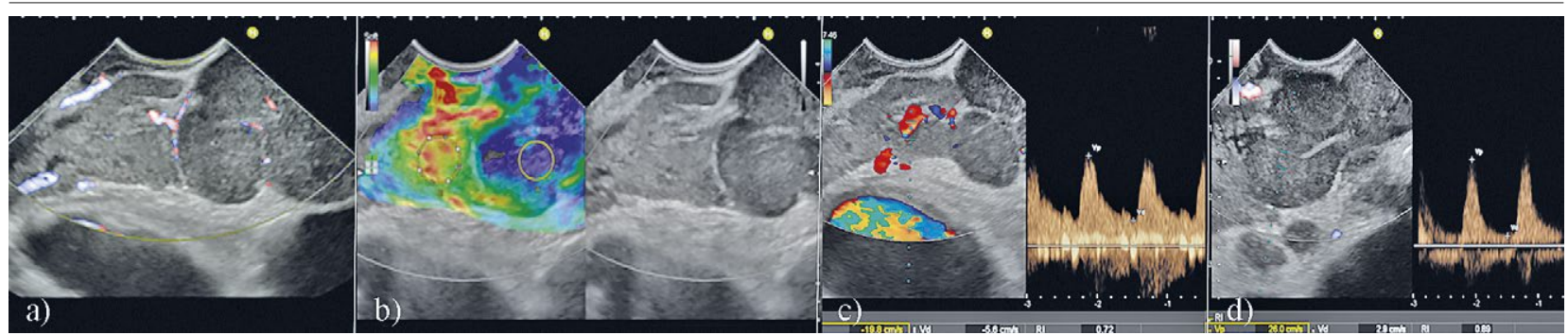

Fig 9. Patient presents with a mass lesion of the right lung, suspicious for lung cancer. EUS is performed for mediastinal LN staging. Several enlarged LNs are found at LN station 7, which differ in echopattern (a; left: medium echogenicity, echogenic hilum; right: low echogenicity, no hilum). With strain elastography, the left node exhibits a soft elastography pattern, whereas the right LN is homogeneously blue (stiff, Strain Ratio compared to the left LN was 78.66) (b). RI of hilar vessels of the left LN is within normal range (c; 0.72), whereas the RI of the right LN is high (d; 0.89). EUS-guided sampling of the right LN was performed with diagnosis of LN metastasis of squamous cell cancer. Take home message: the presence of enlarged hypoechoic LNs with loss of hilar structure, high stiffness with elastography and high RI with Doppler analysis are eye-catching features of LN cancer metastasis.

\section{"Eye-catching features of malignant lymphadenopathy - lymphoma"}

There are different sonographic patterns of peripheral LNs suggesting malignant lymphoma (fig 10). Variably configured hyperechoic areas within a LN are almost exclusively seen in chronic lymphocytic leukemia (fig 11). Particularly, in the case of aggressive lymphomas, hyperechoic infiltrating growth can be observed with destruction of LN architecture (fig 12).

Enlarged LNs of the neck and mediastinum in combination with typical symptoms are indicative of Hodgkin's disease [9] (fig 13). "Potato sac-like" lymphoma can be observed in both indolent and aggressive lymphomas (fig 14). Large "bulky" lymphatic masses surrounding the mesenteric vessels are typical of lymphoma [9] (fig 15). Occasionally, lymphoma masses may present with tumour associated complications that need further sonographic evaluation to establish a final diagnosis (fig 16). In all cases of suspected lymphoma, histological confirmation of diagnosis is absolutely necessary $[9,20]$.

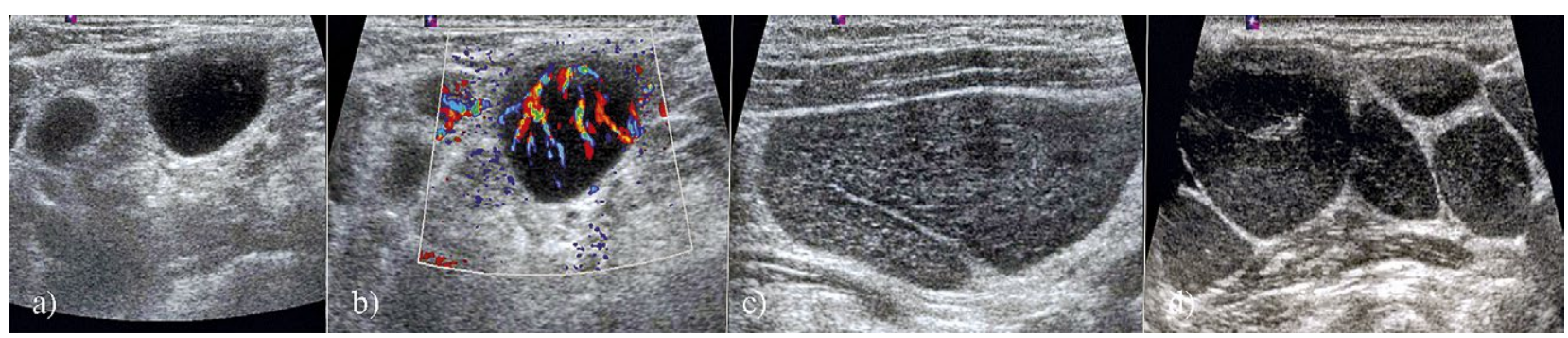

Fig 10. Different B-mode and CDS patterns of malignant lymphoma. Nearly cystic appearance of LNs and hypervascularization on CDS: aggressive NHL $(a, b)$. A marked enlarged, oval-shaped and hypoechoic LN with granular pattern and still visible thin hyperechoic hilum (c). A conglomerate of hypoechoic LNs with smooth delineation join together like a stonewall (d). Take home message: the presence of nearly cystic LNs, the presence of enlarged hypoechoic LNs with granular pattern and conglomerates of LNs like a stonewall are eye-catching features for lymphoma.

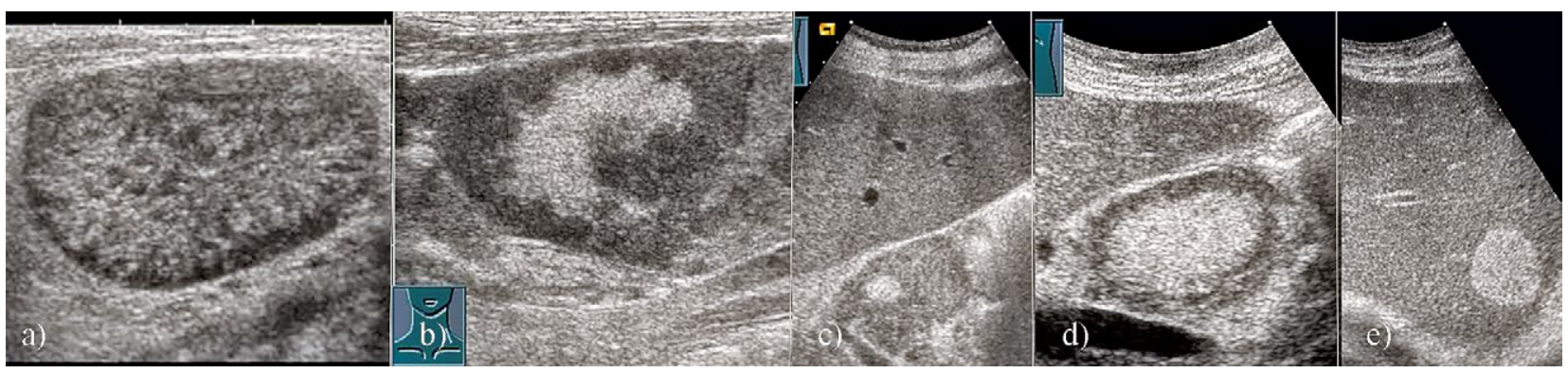

Fig 11. Pattern of lymphoma morphology in patients with chronic lymphocytic leukemia (CLL). Mesh-like hyperechoic infiltration of a LN (a). Focal round hyperechoic areas in the enlarged LNs (b,c,d) and hyperechoic round splenic lesion (e). Take home message: the presence of focal enlarged pathological LNs with hyperechoic round splenic lesion is an eye-catching feature for CLL. 

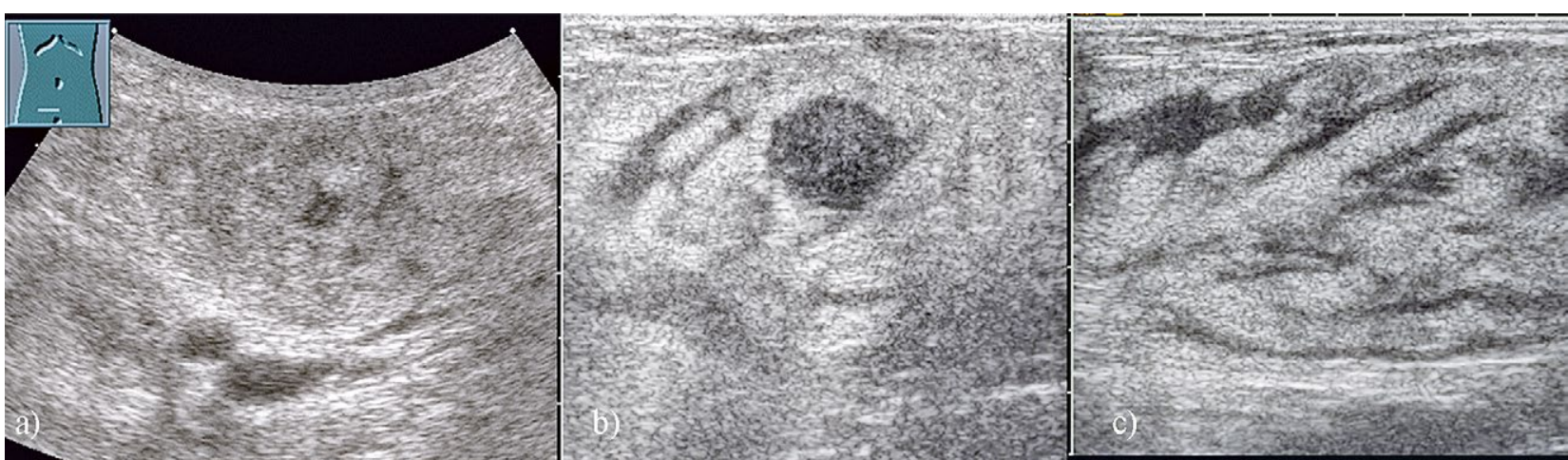

Fig 12. Patient presents with a palpable right sided inguinal tumour. B-mode imaging shows an oval, fat-like soft mass irregular delineated (a). Hyperechoic and hypoechoic areas can be differentiated in high-resolution sonography (b). In individual areas the tumour appears to have a folded structure (c). The US guided core needle biopsy revealed the diagnosis of an aggressive lymphoma. Take home message: the presence of enlarged LNs with hyperechoic and hypoechoic areas and fold structure are eye-catching features for aggressive lymphoma.

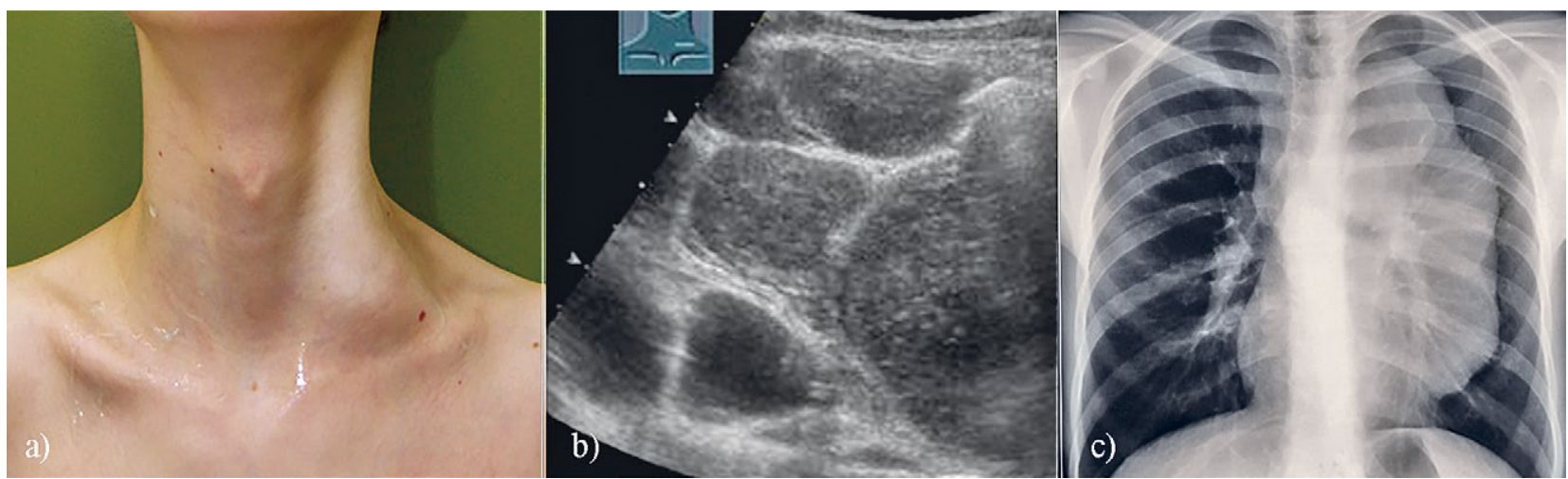

Fig 13. Patient with B-symptoms and a palpable tumour on the left side of the neck (a). B-mode imaging presents multiple enlarged hypoechoic LNs (b). In the chest X-ray, a pronounced mediastinal tumour was observed (c). The US guided core needle biopsy revealed the diagnosis of Hodgkin's disease (d). Take home message: after exclusion of an acute Ebstein Barr Virus (EBV) infection, the presence of multiple huge enlarged pathological hypoechoic LNs unilaterally in the neck is an eye-catching feature for Hodgkin's disease.

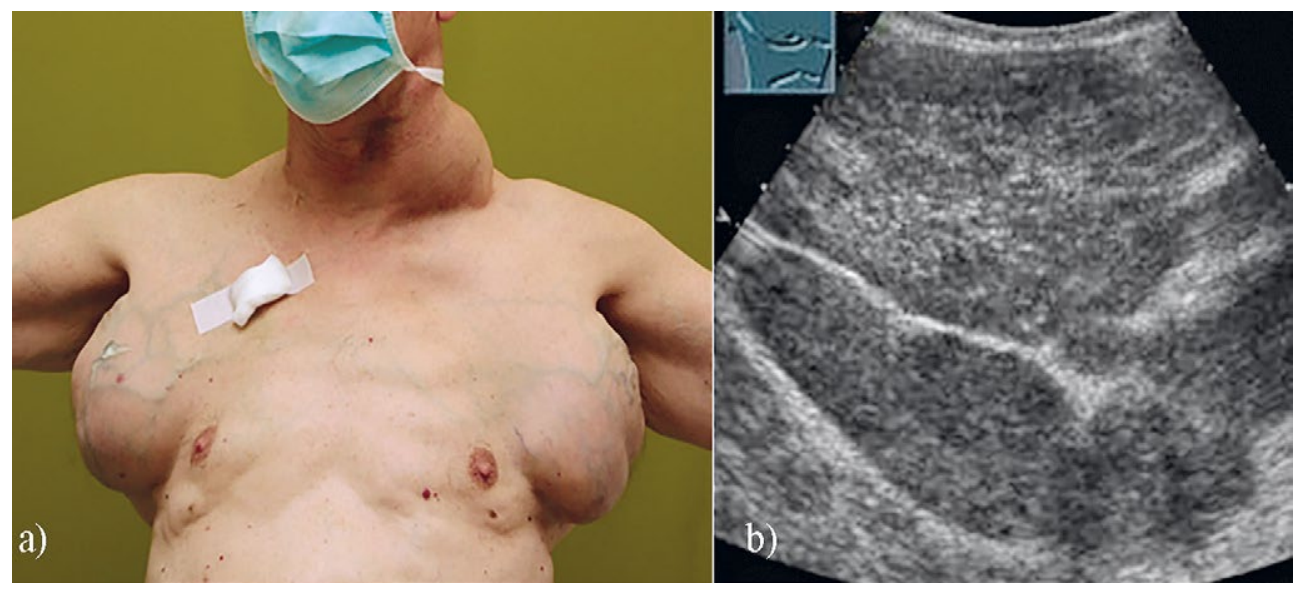

Fig 14. Patient with CLL presents with huge palpable LNs present for years but now growing in all areas (a). B-mode imaging presents the CLL-characteristic "potato sac-like" lymphoma packets (b). Take home message: the presence of "potato sac-like" multiple enlarged LNS is an eye-catching feature for lymphoma. 


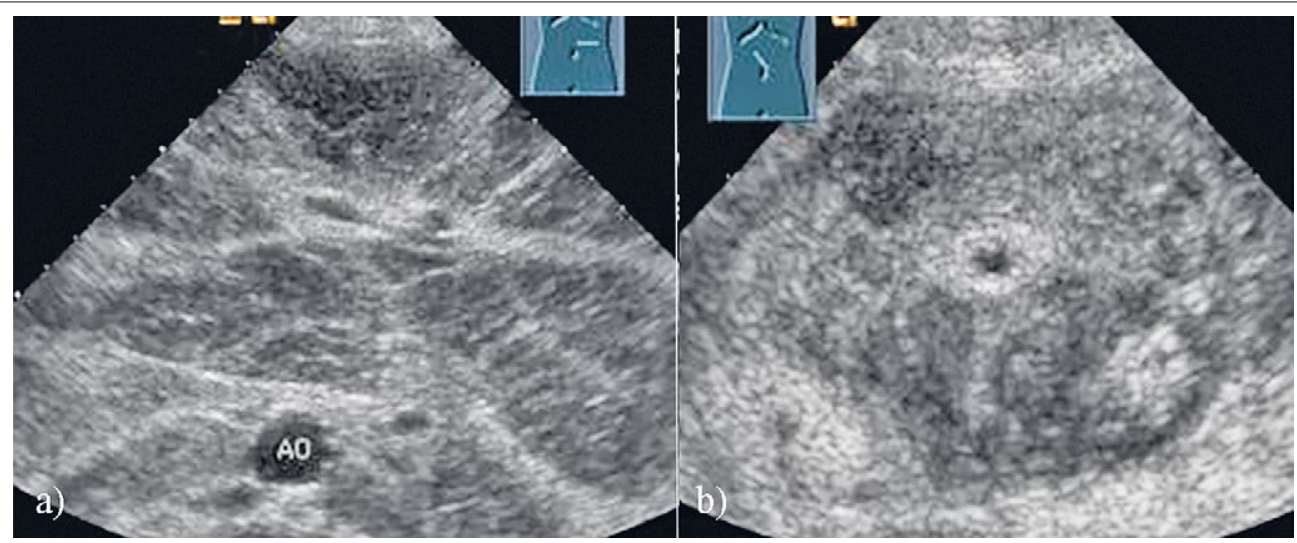

Fig 15. Patients with CLL and patterns of massive mesenteric and para-aortic lymphoma involvement. Confluent hypoechoic LNs located around the mesenteric vessels (a). Nearly homogeneous complex mesenteric tumour formation (b). Take home message: the presence of massive enlarged homogeneous hypoechoic LNs around the mesenteric vessels is an eye-catching feature for mesenteric lymphoma infiltration.

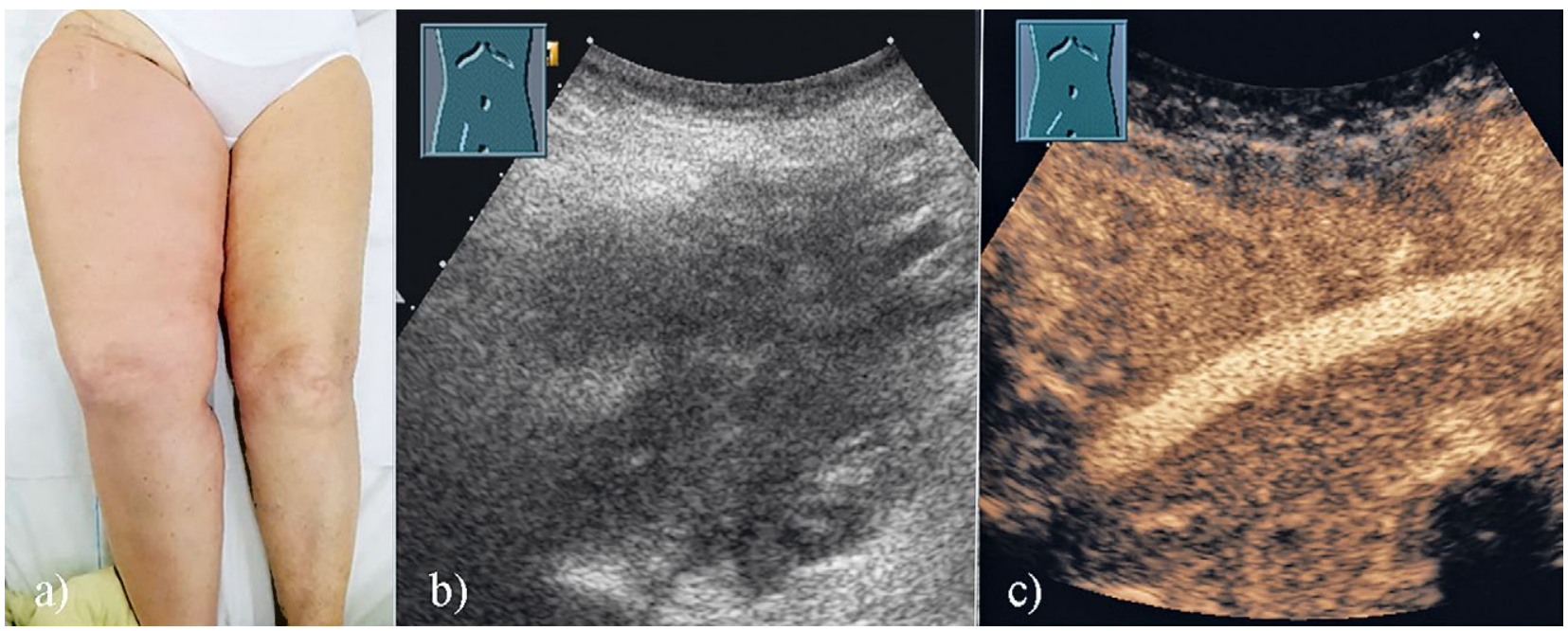

Fig 16. Patient presents with a massive right-sided leg swelling. Diagnosis of deep vein thrombosis was already established and anticoagulation treatment initiated (a). B-mode imaging shows a large hypoechoic mass in the area of the right pelvis (b). On CEUS, marked enhancement of tumour tissue was observed. No venous flow was seen (c). The US guided core needle biopsy revealed the diagnosis of Hodgkin's disease. Take home message: the presence of large hypoechoic LNs in the pelvis with marked enhancement on CEUS is an eye-catching feature for malignant lymphoma.

\section{Conclusion}

Detailed diagnostic characterisation of lymph nodes is a domain of multiparametric ultrasound. Distinct patterns of ultrasound morphology are decisive but the combination of clinical features and sonographic images are the key to diagnosis. The interpretation of enlarged LNs requires a high clinical and sonographic competence. To guide clinical management in cases of suspected lymphoma or metastasis, ultrasound-guided biopsy is essential for histological confirmation of diagnosis and for specific immunohistochemical and molecular characterization.

\section{Conflict of interest: none}

Acknowledgement: The authors thank the Bad Mergentheimer Leberzentrum e.V. for support.

\section{References}

1. Tschammler A, Beer M, Hahn D. Differential diagnosis of lymphadenopathy: power Doppler vs color Doppler sonography. Eur Radiol 2002;12:1794-1799.

2. Tschammler A, Ott G, Schang T, Seelbach-Goebel B, Schwager K, Hahn D. Lymphadenopathy: differentiation of benign from malignant disease--color Doppler US 
assessment of intranodal angioarchitecture. Radiology 1998;208:117-123.

3. Tschammler A, Wirkner H, Ott G, Hahn D. Vascular patterns in reactive and malignant lymphadenopathy. Eur Radiol 1996;6:473-480.

4. Tschammler A, Knitter J, Wittenberg G, Krahe T, Hahn D. Quantification of lymph node perfusion using color duplex ultrasonography. Rofo 1995;163:203-209.

5. Tschammler A, Gunzer U, Reinhart E, et al. The diagnostic assessment of enlarged lymph nodes by the qualitative and semiquantitative evaluation of lymph node perfusion with color-coded duplex sonography. Rofo 1991;154:414418.

6. Rettenbacher T. Sonography of peripheral lymph nodes part 2: Doppler criteria and typical findings of distinct entities. Ultraschall Med 2014;35:10-27.

7. Rettenbacher T. Sonography of peripheral lymph nodes part 1: normal findings and B-image criteria. Ultraschall Med 2010;31:344-362.

8. Cui XW, Hocke M, Jenssen C, et al. Conventional ultrasound for lymph node evaluation, update 2013. Z Gastroenterol 2014;52:212-221.

9. Trenker C, Gorg C, Jenssen C, Klein S, Neubauer A, Dietrich CF. The role of abdominal ultrasound in hematological diseases. Z Gastroenterol 2018;56:1063-1076.

10. Dong Y, Jurgensen C, Puri R, et al. Ultrasound imaging features of isolated pancreatic tuberculosis. Endosc Ultrasound 2018;7:119-127.

11. Barreiros AP, Braden B, Schieferstein-Knauer C, Ignee A, Dietrich CF. Characteristics of intestinal tuberculosis in ultrasonographic techniques. Scand J Gastroenterol 2008;43:1224-1231.

12. Dietrich CF, Brunner V, Lembcke B. Intestinal ultrasound in rare small and large intestinal diseases. Z Gastroenterol 1998;36:955-970.

13. Dietrich CF, Lembcke B, Jenssen C, Hocke M, Ignee A, Hollerweger A. Intestinal ultrasound in rare gastrointestinal diseases, update, part 1. Ultraschall Med 2014;35:400421.

14. Dietrich CF, Lembcke B, Jenssen C, Hocke M, Ignee A, Hollerweger A. Intestinal Ultrasound in Rare Gastrointestinal Diseases, Update, Part 2. Ultraschall Med 2015;36:428456.

15. Dietrich CF, Hollerweger A, Dirks K, et al. EFSUMB Gastrointestinal Ultrasound (GIUS) Task Force Group: Celiac sprue and other rare gastrointestinal diseases ultrasound features. Med Ultrason 2019;21:299-315.

16. Zhou LQ, Wu XL, Huang SY, et al. Lymph Node Metastasis Prediction from Primary Breast Cancer US Images Using Deep Learning. Radiology 2020;294:19-28.

17. Jiang M, Li C, Tang S, et al. Nomogram Based on ShearWave Elastography Radiomics Can Improve Preoperative Cervical Lymph Node Staging for Papillary Thyroid Carcinoma. Thyroid 2020 Mar 11. doi:10.1089/thy.2019.0780.

18. Zhou LQ, Li P, Cui XW, Dietrich CF. Ultrasound nanotheranostics in fighting cancer: Advances and prospects. Cancer Lett 2020;470:204-219.
19. Li J, Chen M, Cao CL, et al. Diagnostic Performance of Acoustic Radiation Force Impulse Elastography for the Differentiation of Benign and Malignant Superficial Lymph Nodes: A Meta-analysis. J Ultrasound Med 2020;39:213222.

20. Trenker C, Gorg C, Jenssen C, et al. Ultrasound in oncology, current perspectives. Z Gastroenterol 2017;55:10211037.

21. Monig SP, Zirbes TK, Schroder W,et al. Staging of gastric cancer: correlation of lymph node size and metastatic infiltration. AJR Am J Roentgenol 1999; 173:365-367.

22. Goerg C, Schwerk WB. Sonographic staging of gastrointestinal lymphoma. Bildgebung 1990;57:21-23.

23. Jenssen C, Dietrich CF, Burmester E. Malignant neoplasias of the gastrointestinal tract--endosonographic staging revisited. Z Gastroenterol 2011;49:357-368.

24. Ahuja A, Ying M. Sonographic evaluation of cervical lymphadenopathy: is power Doppler sonography routinely indicated? Ultrasound Med Biol 2003;29:353-359.

25. Ahuja A, Ying M, Yuen YH, Metreweli C. Power Doppler sonography to differentiate tuberculous cervical lymphadenopathy from nasopharyngeal carcinoma. AJNR Am J Neuroradiol 2001;22:735-740.

26. Ahuja AT, Ying M. Sonographic evaluation of cervical lymph nodes. AJR Am J Roentgenol 2005;184:1691-1699.

27. Hobbs SK, Monsky WL, Yuan F, et al. Regulation of transport pathways in tumor vessels: role of tumor type and microenvironment. Proc Natl Acad Sci U S A 1998;95:46074612.

28. Leboulleux S, Girard E, Rose M, et al. Ultrasound criteria of malignancy for cervical lymph nodes in patients followed up for differentiated thyroid cancer. J Clin Endocrinol Metab 2007;92:3590-3594.

29. Rofstad EK, Tunheim SH, Mathiesen B, et al. Pulmonary and lymph node metastasis is associated with primary tumor interstitial fluid pressure in human melanoma xenografts. Cancer Res 2002;62:661-664.

30. Taghian AG, Abi-Raad R, Assaad SI, et al. Paclitaxel decreases the interstitial fluid pressure and improves oxygenation in breast cancers in patients treated with neoadjuvant chemotherapy: clinical implications. J Clin Oncol 2005;23:1951-1961.

31. Lunt SJ, Kalliomaki TM, Brown A, Yang VX, Milosevic M, Hill RP. Interstitial fluid pressure, vascularity and metastasis in ectopic, orthotopic and spontaneous tumours. BMC Cancer 2008;8:2.

32. Hocke M, Menges M, Topalidis T, Dietrich CF, Stallmach A. Contrast-enhanced endoscopic ultrasound in discrimination between benign and malignant mediastinal and abdominal lymph nodes. J Cancer Res Clin Oncol 2008;134:473480 .

33. Chang DB, Yuan A, Yu CJ, Luh KT, Kuo SH, Yang PC. Differentiation of benign and malignant cervical lymph nodes with color Doppler sonography. AJR Am J Roentgenol 1994; 162:965-968.

34. Wu CH, Chang YL, Hsu WC, Ko JY, Sheen TS, Hsieh FJ. Usefulness of Doppler spectral analysis and power Doppler 
sonography in the differentiation of cervical lymphadenopathies. AJR Am J Roentgenol 1998;171:503-509.

35. Ying M, Ahuja A, Brook F. Repeatability of power Doppler sonography of cervical lymph nodes. Ultrasound Med Biol 2002;28:737-744.

36. Dietrich CF, Hocke M, Jenssen C. Ultrasound for abdominal lymphadenopathy. Dtsch Med Wochenschr 2013;138:10011018.

37. Demharter J, Muller P, Wagner T, Schlimok G, Haude K, Bohndorf K. Percutaneous core-needle biopsy of enlarged lymph nodes in the diagnosis and subclassification of malignant lymphomas. Eur Radiol 2001;11:276-283.
38. Tzankov A, Heiss S, Ebner S, et al. Angiogenesis in nodal B cell lymphomas: a high throughput study. J Clin Pathol 2007;60:476-482.

39. Farinha P, Kyle AH, Minchinton AI, Connors JM, Karsan A, Gascoyne RD. Vascularization predicts overall survival and risk of transformation in follicular lymphoma. Haematologica 2010;95:2157-2160.

40. Cardesa-Salzmann TM, Colomo L, Gutierrez G, et al. High microvessel density determines a poor outcome in patients with diffuse large B-cell lymphoma treated with rituximab plus chemotherapy. Haematologica 2011;96:9961001. 